\title{
Sensibilidade Antimicrobiana de Amostras de Escherichia coli Isoladas de Leitões com Diarréia Após o Desmame.
}

\author{
${\text { Benito Guimarães de } \text { Brito }^{1 *} \text { e Kelly Cristina Tagliari }}^{2}$. \\ ${ }^{1}$ Departamento de Medicina Veterinária Preventiva, Centro de Ciências Agrárias, Universidade Estadual de \\ Londrina, Caixa Postal 6001, CEP: 86.051-970, Londrina - PR, Brasil. ${ }^{2}$ Departamento de Microbiologia, Centro \\ de Ciências Biológicas, Universidade Estadual de Londrina, Caixa Postal 6001, CEP: 86.051-970, Londrina-PR, \\ Brasil
}

\begin{abstract}
The main infectious agent responsible by post-weaning diarrhoea in piglets is the Escherichia coli and the therapy by drugs is used for control of this disease. The antimicrobial sensitivity of 66 strains of Escherichia coli isolated from piglets with post-weaning diarrhoea was studied. The results show high resistance at four drugs (streptomycin, sulfonamide, tetracycline and chloramphenicol). Gentamycin and neomycin were the best antimicrobial in the inhibition of strains of Escherichia coli, isolated from piglets with post-weaning diarrhoea, in the southwest of Paraná.
\end{abstract}

Key words: $E$. coli, antimicrobial sensibility, drugs, piglets, diarrhoea.

\section{INTRODUÇÃO}

As diarréias são freqüentes nas criações de suínos no Estado do Paraná, Brasil, e provocam grandes prejuízos econômicos (Brito et al, 1994 e Brito et al, 1995). Na maioria das vezes, estas diarréias ocorrem de 3 a 10 dias após o desmame (Hampson, 1994). Vários fatores de risco contribuem para a ocorrência das diarréias, podendo ser citados: ausência do vazio sanitário, alta lotação das instalações, além de outras enfermidades intercorrentes (Silva et al, 1999). A Escherichia coli é o principal agente infeccioso envolvido nas diarréias dos leitões após o desmame. Estudo epidemiológico realizado no sudoeste do Paraná por alguns autores (Brito et al, 1996) identificou E. coli como o principal agente etiológico das diarréias dos leitões após o desmame, sendo responsável por $37,9 \%$ dos 145 casos clínicos de diarréia analisados. Dentre as medidas para a redução e controle desta enfermidade encontra-se a antibioticoterapia (Glattleider, 1993). Em virtude da diversidade de comportamento de E. coli frente aos antimicrobianos, principalmente pelo uso de subdoses de antibióticos e pela fácil transferência da resistência através de plasmídios entre amostras bacterianas (Boni \& Sacchi, 1989), é importante o conhecimento do padrão de sensibilidade antimicrobiana desta bactéria para uma efetiva redução do quadro clínico. Trabalhos de sensibilidade aos antibióticos têm sido realizados com resultados variáveis (Barcellos et al, 1977; Wilson, 1981 e Borowski et al, 1993). O objetivo deste trabalho foi determinar a sensibilidade antimicrobiana e os principais perfis de resistência de amostras de E. coli, isoladas de leitões recém-desmamados com diarréia, da região sudoeste do Paraná.

\section{MATERIAL E MÉTODOS}

Foram estudadas sessenta e seis amostras de $E$. coli, isoladas de 66 leitões com diarréia entre 0 a 14 dias após o desmame, de 30 granjas da região sudoeste do Paraná. As amostras foram isoladas a partir de fezes diarréicas cultivadas por 18 horas a $37^{\circ} \mathrm{C} \mathrm{em}$ meios de ágar sangue e ágar Mac Conkey. A identificação foi realizada através das características morfotintoriais e provas bioquímicas (Cowan, 1975). As amostras

* Author para correspondência 
foram avaliadas quanto a resistência aos diversos antimicrobianos através da técnica de difusão do antibiótico impregnado em discos de papel filtro, em ágar Mueller-Hinton (Barry \& Thorwsberry, 1985), usando os seguintes antimicrobianos: gentamicina $(10 \mu \mathrm{g})$, neomicina $(30 \mu \mathrm{g})$, colistina $(10 \mu \mathrm{g})$, ácido nalidíxico $(30 \mu \mathrm{g})$, kanamicina $(30 \mu \mathrm{g})$, nitrofurantoína $(300 \mu \mathrm{g})$, trimetoprim+ sulfamethoxazole $(25 \mu \mathrm{g})$, estreptomicina (10 $\mu \mathrm{g})$, sulfonamidas $(300 \mu \mathrm{g})$, tetraciclina $(30 \mu \mathrm{g})$ e cloranfenicol $(30 \mu \mathrm{g})$. Na Tabela I constam os parâmetros de interpretação utilizados para avaliação dos resultados dos halos de inibição (Barry \& Thorwsberry, 1985). A seleção dos discos baseou-se no critério da disponibilidade comercial dos princípios ativos, utilizados no controle da diarréia pós desmame causada por E. coli.

\section{RESULTADOS E DISCUSSÃO}

$\mathrm{Na}$ Tabela I estão os resultados dos antibiogramas realizados com as 66 amostras de E. coli. Nos testes efetuados, os antimicrobianos que apresentaram melhor resultado ( $>80 \%$ de amostras sensíveis) foram gentamicina, neomicina e ácido nalidíxico. Os antimicrobianos kanamicina, colistina, nitrofurantoína, trimetoprim+sulfamethoxazole, obtiveram resultados intermediários (50 a $80 \%$ de amostras sensíveis), enquanto que estreptomicina, sulfonamidas, tetraciclinas e cloranfenicol foram menos efetivos $(<30 \%$ de amostras sensíveis).

Tabela I. Sensibilidade de 66 amostras de Escherichia coli isoladas de leitões recém-desmamados com diarréia, frente aos antimicrobianos.

\begin{tabular}{|c|c|c|c|c|c|}
\hline \multirow[t]{2}{*}{ ANTIMICROBIANOS } & \multicolumn{3}{|c|}{$\begin{array}{l}\text { HALO DE INIBIÇÃO DE } \\
\text { CRESCIMENTO (mm) }\end{array}$} & \multicolumn{2}{|c|}{$\begin{array}{l}\text { AMOSTRAS } \\
\text { SENSÍVEIS }\end{array}$} \\
\hline & RES & INT & SENS & $\mathrm{N}^{\circ}$ & $\%$ \\
\hline GENTAMICINA & 12 & $13-14$ & 15 & 62 & 94 \\
\hline NEOMICINA & 12 & $13-16$ & 17 & 58 & 88 \\
\hline ÁCIDO NALIDÍXICO & 13 & $14-18$ & 19 & 54 & 82 \\
\hline KANAMICINA & 13 & $14-17$ & 18 & 52 & 79 \\
\hline COLISTINA & 08 & $09-10$ & 11 & 50 & 76 \\
\hline NITROFURANTOÍNA & 14 & $15-16$ & 17 & 48 & 70 \\
\hline TRIMETOPRIM+SULFAMETHOZAXOLE & 10 & $11-15$ & 16 & 34 & 52 \\
\hline ESTREPTOMICINA & 11 & $12-14$ & 15 & 19 & 29 \\
\hline SULFONAMIDAS & 12 & $13-16$ & 17 & 14 & 21 \\
\hline TETRACICLINA & 14 & $15-18$ & 19 & 13 & 20 \\
\hline CLORANFENICOL & 12 & $13-17$ & 18 & 05 & 08 \\
\hline
\end{tabular}

Res: resistente.

Int: intermediário.

Sen: sensível.

As amostras de E. coli isoladas de suínos, no Canadá e na Bélgica (Gannon et al, 1988 e Pohl et al, 1991), foram sensíveis à gentamicina e neomicina, o que está de acordo com os dados do nosso trabalho.
Os níveis de resistência aos antimicrobianos das amostras foram altos (Figura 01), o que coincide com relatos de levantamentos semelhantes realizados em outros países (Liven, 1979; Coates \& Hoopes, 1980; Larsen \& Sogard, 1981 e Libal \& Gates, 1982). 


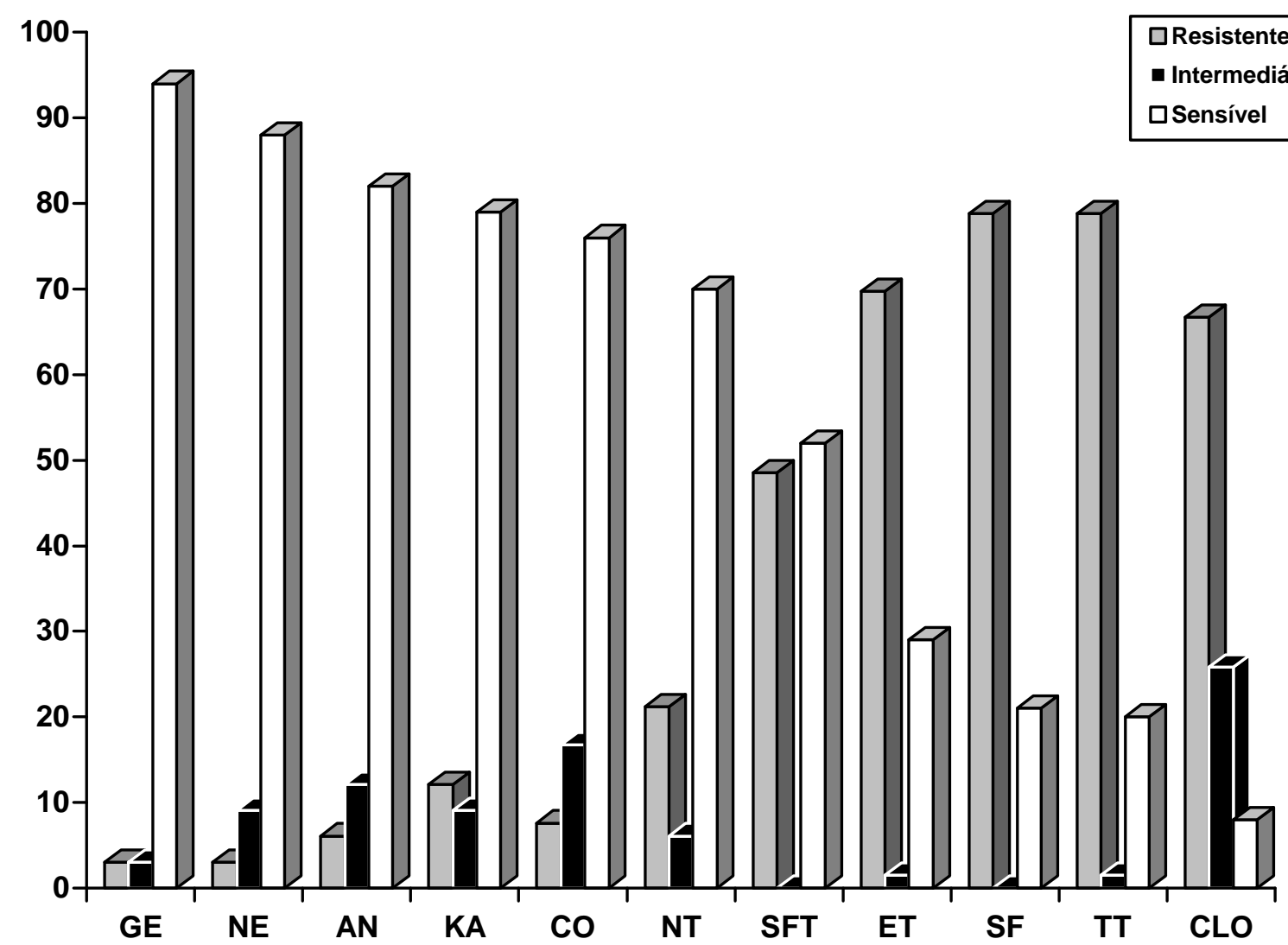

Figura 01. Percentual do comportamento de amostras de Escherichia coli isoladas de suínos com diarréia, frente aos antimicrobianos: gentamicina (GE), neomicina (NE), ácido nalidíxico (AN), kanamicina (KA), colistina (CO), nitrofurantoína (NT), trimetoprim + sulfamethoxazole (SFT), estreptomicina (ET), sulfonamidas (SF), tetraciclina (TT) e cloranfenicol (CLO).

$\mathrm{O}$ alto percentual de resistência à tetraciclina observado neste experimento também foi observado por vários autores (Borowski et al, 1993; Garcia et al, 1987; Awad-Masalmeh et al, 1992 e Sacchi \& Boni, 1989), no Brasil, Venezuela, Áustria e Itália, respectivamente. Alguns autores (Borowski et al, 1993), também encontraram alto percentual de amostras de E. coli resistentes ao cloranfenicol e sulfonamidas oriundas de leitões com diarréia da região de Santa Rosa no Rio Grande do Sul. A alta resistência das cepas de E. coli aos antimicrobianos pode ser devida ao uso inadequado destas drogas em medicações preventivas na ração dos animais.
A Tabela II mostra que o padrão de resistência mais freqüentemente observado, verificado em $11 \quad(16,7 \%)$ das amostras, foi estreptomicina.cloranfenicol.tetraciclina. trimetoprim+sulfamethoxazole.sulfonamidas.

Entre as amostras estudadas verificou-se que somente $4(6,1 \%)$ foram sensíveis a todas as drogas testadas. Nas demais, foram enquadradas 47,2\% das amostras em 6 padrões de resistência, os quais apresentavam resistência a 4 ou mais entre 11 produtos testados.

Tabela II. Perfil de resistência de amostras de Escherichia coli isoladas de leitões recém-desmamados com diarréia, frente aos antimicrobianos. 


\begin{tabular}{l|c|c}
\hline PERFIL DE RESISTÊNCIA ANTIMICROBIANA & \multicolumn{2}{|c}{$\begin{array}{c}\text { FREQÜÊNCIA } \\
\text { N } \mathbf{\%}^{\circ}\end{array}$} \\
\hline $\begin{array}{l}\text { Estreptomicina.Cloranfenicol.Tetraciclina.Trimetoprim+Sulfamethoxazole. } \\
\text { Sulfonamidas }\end{array}$ & 11 & 16,7 \\
\hline $\begin{array}{l}\text { Estreptomicina.Cloranfenicol.Nitrofurantoína.Tetraciclina.Trimetoprim+ } \\
\text { Sulfamethoxazole.Sulfonamidas }\end{array}$ & 05 & 7,6 \\
\hline Estreptomicina.Kanamicina.Cloranfenicol.Tetraciclina.Sulfonamidas & 04 & 6,1 \\
\hline Cloranfenicol.Tetraciclina.Trimetoprim+Sulfomethoxazole.Sulfonamidas & 04 & 6,1 \\
\hline Estreptomicina.Tetraciclina.Trimetoprim+Sulfomethoxazole.Sulfonamidas & 04 & 6,1 \\
\hline Estreptomicina.Tetraciclina.Cloranfenicol.Sulfonamidas & 03 & 4,6 \\
\hline Tetraciclina.Cloranfenicol & 02 & 3,0 \\
\hline Estreptomicina.Sulfonamidas & 02 & 3,0 \\
\hline Estreptomicina.Sulfonamidas.Cloranfenicol & 02 & 3,0 \\
\hline Colistina & 02 & 3,0 \\
\hline Estreptomicina.Sulfonamidas.Tetraciclina & 02 & 3,0 \\
\hline Nitrofurantoína.Estreptomicina.Sulfonamidas.Tetraciclina & 02 & 3,0 \\
\hline Tetraciclina.Trimetoprim+Sulfomethoxazole.Sulfonamidas & 02 & 3,0 \\
\hline Outros padrões de resistência & 17 & 25,8 \\
\hline Sem resistência às drogas & 04 & 6,1 \\
\hline Total & 66 & 100 \\
\hline
\end{tabular}

\section{CONCLUSÕES}

Foi observada alta resistência das amostras estudadas à estreptomicina, sulfonamidas, tetraciclina e cloranfenicol. A variabilidade de resistência encontrada entre as amostras neste estudo pode dificultar a terapêutica antimicrobiana bem como o monitoramento de resistência.

Os antimicrobianos gentamicina e neomicina foram os mais eficientes na inibição do crescimento das amostras de E. coli isoladas de suínos com diarréia, da região sudoeste do Paraná.

\section{AGRADECIMENTOS}

Ao Instituto Agronômico do Paraná (IAPAR), pelo financiamento do projeto.

\section{REFERÊNCIAS BIBLIOGRÁFICAS}

Awad-Masalmeh, M.; Hinterdorfer, F.; El Hamaki-Jelinek, $\mathrm{H}$. et al. Serotypes, $\mathrm{K}_{88}$ antigen, sensitivity to colicins and to chemotherapeutics of enteropathogenic $E$. coli isolated at two Austrian investigation centres between 1988 and 1990. In: CONGRESS INTERNATIONAL PIG VETERINARY SOCIETY, v.12. Hague: IPVS, 1992. Proceedings... p.248.

Barcellos, D. E. S. N.; Ribeiro, L. A. O.; Warth, W. Colibacilose suína: sensibilidade aos antibióticos de amostras patogênicas de $E$. coli isoladas de suínos. Bol. IPVDF, v.4, p.73-8, 1977.

Barry, A. L.; Thorwsberry, C. In: Manual of Clinical Microbiology, v.4. 1985. p.978-87.

BONI, P.; SACCHI, C. L'antibiotico resistenza nella specie suina: concetti generalli. Selezione Veterinaria, v.30, n.4, p.627-34, 1989.

Borowski, S. M.; Barcellos, D. E. S. N.; Stepan, A. L. et al. Sensibilidade a antimicrobianos de amostras de E. coli isoladas de suínos recentemente desmamados. In: CONGRESSO BRASILEIRO DE VETERINÁRIOS ESPECIALISTAS EM SUÍNOS, v.6. Goiânia : ABRAVES, 1993. Anais... p.81.

Brito, B. G.; Bertol, T. M.; Chiapetti, D. Ocorrência de diarréias em leitões recentemente desmamados e sua influência no ganho de peso. In: REUNIÃO ANUAL DA SOCIEDADE BRASILEIRA DE ZOOTECNIA, v.31. Maringá: SBZ, 1994. Anais... p.594. 
Brito, B. G.; Filippsen, L. F.; Mores, N. Estudo ecopatológico da diarréia pós-desmame em granjas suinícolas do sudoeste do Paraná. Ciência Rural, v.25, n.3, p.431-5, 1995.

Brito, B. G.; Ramos, A. P.; Linhares, R. E. et al. Etiologia das diarréias de leitões recentemente desmamados nas granjas de suínos do sudoeste do Paraná. In: CONGRESSO BRASILEIRO DE MEDICINA VETERINÁRIA, V.14. Goiânia: SBMV, 1996. Anais... 1996p.200-1.

Coates, S. R.; Hoopes, K. H. Sensitivities of Escherichia coli isolated from bovine and porcine enteric infections to antimicrobial antibiotics. Am. J. Vet. Res., v.141, n.11, p.1882-3, 1980.

Cowan, S. T. Cowan and Steel's Manual for the Identification of Medical Bacteria. 2 ed. Cambridge: Cambridge University. Press, 1975.

Gannon, V. P. J.; Gyles, C.L.; Friendship, R. W. Characteristics of verotoxigenic Escherichia coli from pigs. Canadian Journal of Veterinary Research, v.52, p.331-7, 1988.

Garcia, D.; Cavazza, M. E.; Botero, L. et al. Preliminary characterization of Escherichia coli isolated from pigs with diarrhoea in Venezuela. Vet. Microbiol., v.13, p.47-56, 1987.

Glattleider, D. L. Pathologie digestive du porc en croissance et alimentation. Rec. Med. Vet., v.169, n.8/9, p.719-32, 1993.

Hampson, D. J. Postweaning Escherichia coli diarrhoea in pigs. In: Escherichia coli in domestic animals and humans. CAB International: Oxon, 1994. p.171-91.
Larsen, J. L.; Sogard, H. The susceptibility of enteropathogenic porcine Escherichia coli strains to polymixin and other antibiotics. Nord. Vet. Med., v.33, p.393-402, 1981.

Libal, M.; Gates, C. E. Antimicrobial resistance in Escherichia coli strains isolated from pigs with diarrhoea. JAVMA, v.180, n.83, p.9089, 1982.

Liven, E. Drug resistance in strains of Escherichia coli from the intestinal tract of pigs in Norway. Acta Vet. Scand., v.20, p.258-69, 1979.

Pohl, P.; Verlinden, M.; Lintermans, P.; Van Robaeys, G.; Stockmans, F. Antibiogrammes des entérobactéries pathogènes pour les animaux d'élevage et les pigeons, isolées en Belgique de 1996 à 1990. Annales de Médecine Vétérinaire, v.135, p.101-8, 1991.

Sacchi, C.; Boni, P. L'antibiotico-resistenza nella specie suina: verifica della situazione nella pratica. Selezione Veterinaria, v.30, n.4, p.635-8, 1989.

Silva, C. A.; Brito, B. G. De; Mores, N.; Amaral, A.L. do. Ecopatologia da diarréia pós-desmame em granjas de suínos da região Norte do Paraná. Ciência Rural, v.29, n.1, p.39-43, 1999.

Wilson, M. R. Enteric Colibacillosis. In: Leman, A. D. et al. Diseases of swine. 5 ed. Iowa: State University Press, 1981. p.471-7.

Received: July 03, 1998; Revised: September 09, 1999; Accepted: November 25, 1999. 